\title{
Students' understanding of the concept of the electric field through conversions of multiple representations
}

\author{
Esmeralda Campos $\odot,{ }^{1}$ Genaro Zavala®${ }^{1,2,{ }^{*}}$ Kristina Zuza $\odot,{ }^{3}$ and Jenaro Guisasola ${ }^{3}$ \\ ${ }^{1}$ Tecnologico de Monterrey, Escuela de Ingenieria y Ciencias, \\ E. Garza Sada 2501, Monterrey 64849, Mexico \\ ${ }^{2}$ Universidad Andres Bello, Facultad de Ingenieria, Antonio Varas 810, \\ Providencia, Santiago 7500971, Chile \\ ${ }^{3}$ University of the Basque Country, Donostia Physics Education Research Group and Applied Physics \\ Department, School of Engineering Gipuzkoa (UPV/EHU), Plaza Europa 1, San Sebastian 20018, Spain
}

(Received 23 September 2019; accepted 22 April 2020; published 4 June 2020)

\begin{abstract}
We conducted a study with introductory and upper-division level physics students in a Mexican and a Spanish university to learn how students recognize the main characteristics of the electric field in three of its more widely used representations, namely, algebraic notation, vector field plot, and electric field lines, and how the students do conversions of them. The students' abilities to recognize the three representations of the electric field and do conversions gave insight into their understanding of this concept. We used the theory of registers of semiotic representations as a framework to analyze the data. Our results showed that the direction of the conversion is an essential factor in determining the students' success in performing conversions of electrical field representations. We found close synergy between the vector field plot and the algebraic notation of the electric field. However, we found that the conversions that involve electric field lines do not present synergy. The electric field lines representation is especially difficult for students, both as a source and as a target representation, specifically, the interpretation and representation of the magnitude of the field through the density of field lines. We recommend that teachers and researchers of electricity and magnetism be more conscious of the difficulties that some conversion tasks may present to their students. We specifically invite instructors to be attentive to how they approach the representation of electric field lines and be explicit when performing conversions that involve electric field lines.
\end{abstract}

DOI: 10.1103/PhysRevPhysEducRes.16.010135

\section{INTRODUCTION}

Understanding the concept of field is a crucial step in developing a Maxwellian perspective in the study of electromagnetism. Previous studies have emphasized the importance of adopting a Maxwellian perspective for addressing students' confusion between forces and fields as well as other difficulties related to the understanding of field theory [1-3]. In electricity and magnetism courses at the undergraduate level, field theory plays a significant role in understanding interactions at a distance, which is first introduced through a Newtonian worldview (i.e., using Coulomb's law) and, subsequently, through a Maxwellian viewpoint on the concept of the electric field. Several studies have identified the major difficulties that students have understanding the concept of an electric field [1-6],

*genaro.zavala@tec.mx

Published by the American Physical Society under the terms of the Creative Commons Attribution 4.0 International license. Further distribution of this work must maintain attribution to the author(s) and the published article's title, journal citation, and DOI. such as confusing forces and fields [1,2,7], failing to apply the superposition principle of electric fields [8-12], and misinterpreting the representations of electric fields [11-23].

In physics education research, the role of multiple representations in developing students' problem-solving abilities has been widely studied [24-32]. The literature has focused on representational use for problem solving and its differences between experts and novice users. It has been found that student performance in solving physics problems depends on representational format [24,25] and the particular combination of representations, its relation to the topic, and students' previous knowledge [26]. Students apply different problem-solving strategies that depend on the format in which the problem is represented [27]. It is essential to involve students actively in representational use so they can choose appropriate representations consistently and coordinate their use with other representations [28].

However, the effect of representational use on students' understanding of abstract concepts has not been explored in the context of the electric field. For this, the theory of semiotic representations [33] creates a direct link between the students' abilities to transform semiotic 
representations and their conceptual understanding of the abstract object represented.

In this article, we explore the role that using multiple representations may have on undergraduate students' conceptual understanding of the electric field. For this objective, we focus on the transformations among the three most widely used representations of the electric field in introductory physics textbooks, namely, the algebraic notation, the vector field plot, and the electric field lines diagram. We conducted a study in Mexico and Spain among introductory and upper-division level students enrolled in electricity and magnetism courses. The objective was to explore their abilities to transform between the mentioned representations of the electric field with a set of six openended questions that focus on a conversion task and their explanations. We analyzed the data in light of the primary sources of difficulty expounded by Duval [33], namely, recognition and conversion.

We have organized this article as follows. In Sec. II, we review the main literature that covers the use of representations for problem solving and the conceptual understanding of the electric field and its associated representations. In Sec. III, we introduce Duval's theory of semiotic representations as the theoretical framework, and we define the representations of the electric field that we consider in this study. In Sec. IV, we explain the methodology and data analysis in detail. In Sec. V, we present the results, followed by the discussion in Sec. VI. In Sec. VII, we conclude with a summary of the results and their implications for teaching.

\section{PREVIOUS STUDIES ON CONCEPTUAL UNDERSTANDING OF THE ELECTRIC FIELD AND ITS REPRESENTATION}

\section{A. Conceptual understanding of the electric field}

Students have ontological and epistemological difficulties grasping the concept of the electric field as part of the Maxwellian model. Instead, they tend to stick to a Coulomb perspective and to the Newtonian model of action-at-adistance to explain electrical interactions [1]. They tend to think of the electrical nature of matter as a fluid that moves from one charged body to another [4,34], and they confuse the concept of the electric field with electric force $[1,2,7]$. Students have difficulties changing from the Newtonian (based on Coulomb's law) to the Maxwellian perspective (based on fields) to explain the concept of an electric field, which could be due to an immature understanding of the electric and magnetic field as vector fields [3]. Most students do not have a coherent model for the characteristics of the field, changing back and forth between Newtonian and Maxwellian perspectives [2]. Students often have difficulties relating the concept of an electric field to its effects of charge induction and polarization in matter. For example, they use linear causal reasoning when associating the existence of an electric field with the distribution of charges or to electric current in de circuits. Instead, they should try to understand the macroscopic and microscopic effects [5]. They also fail to consider the conditions of electrostatic equilibrium for charge induction in conductors [6].

Viennot and Rainson [8] found that students fail to apply the superposition principle in isolating materials, and they associate the existence of an electric field to the movement of charges. This idea affects the correct application of the superposition principle [9]. Many students do not differentiate between the contributions of individual charges to the electric field and the net electric field, and some students believe that only the nearest charge contributes to the electric field at a point [10]. Students perform better using the superposition principle for the electric force than for the electric field [11]. Some students think that the electric field produced by charges in a straight line is blocked and that the field cannot be zero if there are only positive charges present [12].

\section{B. Difficulties associated with representations of the electric field}

Rainson, Tranströmer, and Viennot [9] reported that an incorrect interpretation of mathematical relations results in students having difficulties in applying the superposition principle of electric fields. Albe, Venturini, and Lascours [13] found that the use of mathematical formalisms is difficult for students and that they do not relate the concepts with physical models. Even junior engineering students find it complicated to relate mathematics calculations to the physics ideas, to consider spatial situations when performing calculations, and to access the appropriate mathematical tools, which results in several difficulties in upper-division electricity and magnetism $[14,15]$.

Barniol and Zavala [35] studied students' difficulties when using the unit-vector notation and when translating to the graphical representation. Other studies have investigated students' difficulties translating from an algebraic notation to a vector field, precisely, in the dot product and the cross product calculated in electromagnetism [16,17]. Furthermore, Bollen, Van Kampen, Baily, Kelly, and De Cock [36] designed a test to identify students' difficulties in switching between symbolic representation (algebraic), vector field plots, and field lines. They found that students have difficulties with the vector sum and that they have trouble selecting an appropriate coordinate system when they construct mathematical expressions of vector fields themselves [36].

Regarding the difficulties that students have with vector field plot representations, Knight [37] emphasized that introductory physics students need explicit teaching on the use of vectors and practice to familiarize themselves with the representation. Even though many physical quantities are vectors, many students do not associate the representation with the physical concept and its underlying principles. 
For example, after traditional instruction, many students have difficulties understanding the vector nature of Newton's second law, relating forces and movement, and adding or subtracting vectors efficiently [38]. It has also been found that students have difficulties understanding the graphical properties of vectors, such as magnitude and direction, and the components, as well as the procedural properties like sum, negative scalar multiplication, and cross product [35].

The superposition principle may be difficult for students due to a lack of abilities and experience with the graphical representation of vectors. The graphical superposition of vectors is a problem for introductory physics students, even when they undertake the electricity and magnetism courses after having exposure to other vector quantities in mechanics courses [18]. The most common methods that students use to apply vector sum are the head-to-tail, the components method, and the bisection [39]. On the other hand, Gire and Price [19] found that upper-division students studying electricity and magnetism are more familiar with the use of vectors, and the graphical representation gives them the necessary elements to apply the principle of superposition.

We have identified the main difficulties reported in the literature that students have understanding the electric field lines diagram. Students tend to treat electric field lines as if they were real [20], as entities or tubes that transport charge $[21,22]$, and they confuse field lines with trajectories [23]. Also, students have difficulties distinguishing between electric field lines, the electric field produced by a single charge at a point, and the net electric field at that point [12].

The literature reports specific difficulties students have with interpreting the magnitude, direction, and the superposition principle in the electric field lines diagram. They often have difficulties relating the electric field lines diagram with the magnitude of the field [3]; precisely, they do not interpret the electric field lines density as the relative magnitude of the field $[20,40]$. Students think that the curve of the electric field lines gives the direction of the electric field at a position instead of the tangent to the field lines [12]. Also, the literature reports that some characteristics and rules of electric field lines diagrams may create a blocking effect on the correct application of the principle of superposition $[11,40]$.

\section{STUDY}

In physics education research, a few studies acknowledge the difficulties when dealing with semiotic representations [41-44]. Our objective is to investigate students' difficulties in recognizing the characteristics of the electric field (i.e., magnitude and direction) in its three more relevant representations (i.e., algebraic notation, vector field plots, and electric field lines diagrams) and doing conversions between them. We aim to relate their difficulties of recognition and conversion to the conceptual understanding of the electric field at the university level.
Our research questions are

(a) How do students recognize the characteristics of the electric field in different semiotic representations?

(b) How do students convert between different semiotic representations of the electric field?

\section{A. Theoretical framework}

To tackle the research questions, we use Duval's theory of registers of semiotic representations as a theoretical framework to relate the use of multiple representations to conceptual understanding. According to Duval [33], representation is something that stands for something else, either tangible objects or ideas and concepts. Representations of an object can be physical or semiotic. Physical representations are created through physical devices, like photographs or measurements. Semiotic representations use symbols, rules, and associations as tools to represent the object. In natural sciences like physics, chemistry, and biology, objects are often represented with several semiotic systems. The objects of study in natural sciences are directly or indirectly approachable, which allows relating each representation to the object. In contrast, mathematical objects of study are only accessible through semiotic representations [33].

From a cognitive standpoint, mathematical activity resides in the use of semiotic representations that allow the development of mathematical thought [33]. The role of semiotic representations in mathematical cognitive activity creates a paradox because, on the one hand, one can only access the mathematical object through semiotic representations, and, on the other hand, one should never confuse the mathematical object with its representation. For example, students might interpret the arrows that represent a vector with the concept of a vector itself. So the challenge in the learning of mathematics is to dissociate the mathematical object from its representations, which can only be achieved through the use of multiple semiotic representations of the same mathematical object.

The use of multiple semiotic representations of the same mathematical object requires transformations, which can be treatments or conversions [33]. In treatments, the transformation happens within the same semiotic system and depends only on its symbols and rules. Conversion, on the other hand, is the transformation between two semiotic systems without changing the mathematical objects. The conversion task is more complicated than the treatment because it requires the recognition of the same object between two semiotic systems that usually have nothing in common. Thus, the primary sources of difficulty are the recognition of the mathematical object in different semiotic systems and the conversion between them without accessing the mathematical object directly.

In conversion tasks, students may recognize the object in one of the representations, but not as quickly in the other one, which leads to an inappropriate conversion. If the object is not clearly identified in one or several 
representations, it implies a difficulty in the conceptual understanding of the object. It can also be the case that students recognize the object in several representations efficiently and are capable of performing a conversion from one representation to the other but are not able to switch the direction of conversion. In other words, sometimes students can convert from a representation A to a representation B successfully. However, when they are asked to convert from $\mathrm{B}$ to A, the success rate of the task considerably drops [33]. In such a case, it might not be a conceptual difficulty related to recognition, but the difficulty may arise from specific features of the representation systems.

\section{B. Definition of representation systems within the electric field context}

Duval's theory of semiotic representations was developed for the didactics of Mathematics, and it is based on the premise that mathematical objects are not directly accessible. In other natural sciences like physics, chemistry, and biology, the objects of study are accessible through observation by direct or indirect measurements. Some physical quantities are not directly accessible, and therefore may require semiotic representations. The concept of the electric field is a physical quantity that explains electrical interactions; it can only be accessed by indirect measurements (such as the electric current in an electric field mill) and by semiotic representations.

The electric field representation systems that we consider in this study are typically found in many introductory physics textbooks [45-48], i.e., the algebraic notation of the field, the vector plot, and the electric field lines diagram. The three representations are illustrated in Fig. 1. To represent the electric field, the algebraic notation of the field uses vector equations; vector plots use arrows in different points in space, and electric field lines diagrams are a set of curves that give a big picture of the field in space. In the following sections, we define the symbols, rules, and associations of each of these representation systems.

\section{Algebraic notation of electric field}

The algebraic notation requires formulas and vector equations to represent the electric field. The main symbols are numbers and constants (e.g., $\varepsilon_{0}, \pi, \mu_{0}, q$ ), operational symbols (e.g., $=,+,-,>$ ), vector coordinates (e.g., Cartesian: $\hat{i}, \hat{j}, \hat{k}$ ), variables (e.g., Cartesian: $x, y, z$ ), and physical vector quantities in the context of electromagnetic field (e.g., $\vec{E}, \vec{B}, \vec{r}$ ). $\vec{E}$ represents the electric field; the vector coordinates with the + or - symbols represent the direction; numbers, constants, and variables represent the magnitude, and operational symbols represent the superposition principle.

When considering the algebraic notation of the field, it is essential to acknowledge the rules given by the context, since any equation of electric field should respect the physical meaning of Maxwell's equations, that is, the divergence of the electric field denotes the presence of a source of the field, and its curl should be equal to zero in the static case and related to the time variation of the magnetic field in the dynamic case. It is related to a function of electric potential through a negative gradient.

\section{Electric field vector plot}

The electric field vector plot is a set of arrows located at different positions that give a broad idea of the electric field of a system of charges. The symbols are arrows and a coordinate system. The coordinate system represents the positions of space where the electric field is. The length of the arrow represents the magnitude of the field, and the direction and head of the arrow represent the direction of the field at that specific location. Vector addition and subtraction strategies allow applying the principle of superposition graphically. Each arrow represents the electric field at a specific location, which means that all arrows are independent of each other, and they can intersect. The arrows start at the position where the electric field is represented and end with the head of the arrow.

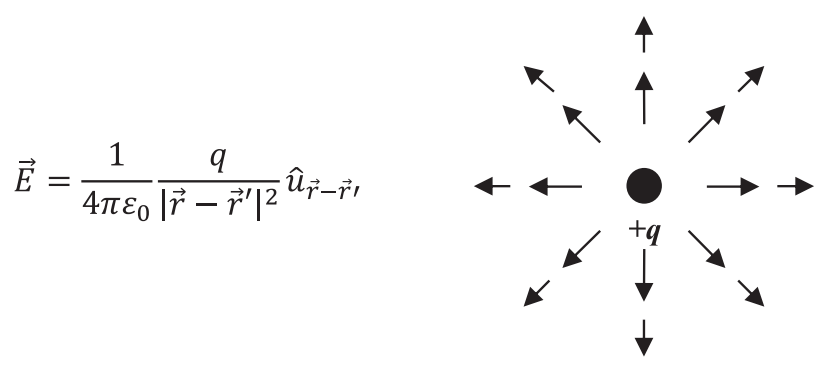

(a) (b)

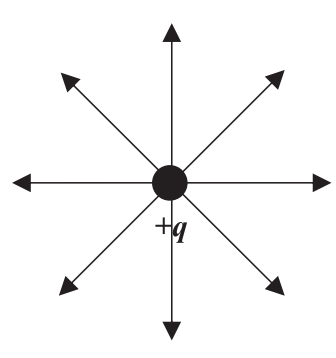

(c)

FIG. 1. The three most common representations of the electric field for a single positive charge. (a) The algebraic notation, which uses algebraic features to represent the magnitude and direction of the field. (b) The vector field plot, which uses arrows in different points in space, and (c) is an electric field lines diagram. 


\section{Electric field lines diagram}

Electric field lines are continuous curves that give an overall visualization of the electric field of a system of charges. The symbols are the curves and arrowheads. The density of field lines (or in two dimensions, the closeness between the field lines) represents the relative magnitude of the field. The arrow's head shows the direction of the field line, and the tangent of the curve at a point shows the direction of the electric field. It is not possible to apply the superposition principle explicitly on the same diagram if the distribution of charges is changed; in that case, it is necessary to construct a new diagram because both the density of lines and the curvature of field lines may change. It is sometimes useful to include other symbols to represent the sources of an electric field (e.g., small circles to represent point charges) [40]. As a rule, field lines start or end necessarily on electric charges, or they extend to infinity for Coulombic fields, while for non-Coulombic fields, there is no start or end. Field lines should never cross since that would imply that the electric field has more than one direction at the intersection.

\section{METHODOLOGY}

\section{A. Instrument}

To achieve the objectives of the investigation, we designed an open-ended questionnaire comprised of six questions that present a conversion task between representations of the electric field. Students are asked to convert between the algebraic notation of the electric field, the vector field plot, and the electric field lines diagram. The objectives of the questions are partly based on the vector fields representations test, an instrument to study students' abilities to translate between algebraic, vector plots, and field lines representations [36], but the physical situations are different because we provide the context of the electric field.

To avoid a possible influence by the way the conversion tasks are presented, we divided the test into two parts, separating the directions of conversion. Figure 2 shows the distribution of the objectives of conversion on each test. The two parts of the test were validated by five researchers in the area of physics education research, who teach introductory physics or electromagnetism courses in the upper division, with consensus on the learning goals of each question. All questions have the objective to study students' ability to recognize the characteristics of the electric field and do conversions between two of three representations, namely, algebraic notation, vector field plots, and electric field lines. For further detail, the two parts of the questionnaire are presented in the Appendix.

The first question (Q1.1 and Q2.1) poses a very large nonconductive plate of height $d$ with uniform volumetric charge density $\rho$ along with the plate. Students are given a three-dimensional sketch of the situation, locating the

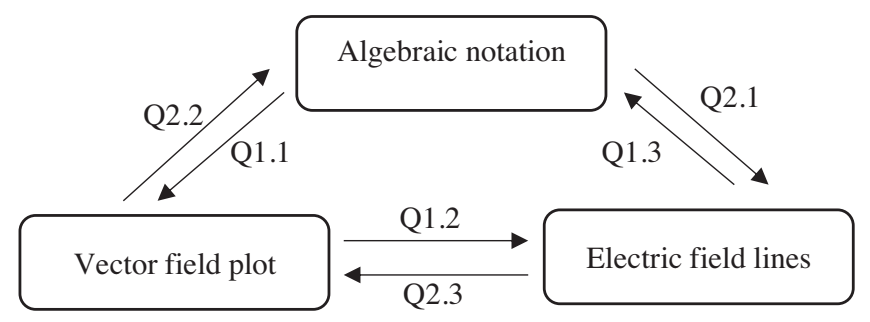

FIG. 2. Distribution of the objectives of conversion in the two parts of the test. The first part is represented by the inside counterclockwise arrows and the second part by the outer, clockwise arrows. The tags correspond to the part of the test (Q1 or Q2) and the question (1,2, or 3) that tackles that specific conversion task.

middle of the plate at the $x-y$ plane, where $z=0$. It then gives the students the electric field created by this plate in an algebraic notation that identifies three regions, being above the plate, inside the plate, and below the plate. In this question, students do not need to perform any computations. They only need to convert from the source representation, i.e., the algebraic notation of the field to a target representation, which is the vector field plot in Q1.1, and an electric field lines diagram in Q2.1. They are given a sketch of a two-dimensional frontal cut of the plate to facilitate their drawing process. They are also asked to explain how their target representation is related to the magnitude and direction of the electric field.

In the second question (Q1.2 and Q2.2), a uniform electric field is shown in a vector field plot representation in an $x-y$ plane. The diagram does not show any sources of the field. The $x-y$ plane is marked with gridlines to allow students to identify the relative size of the vectors. All vectors have the same length and angle with respect to the $x$ and $y$ axes. Students do not need to make any further manipulations to the vectors like addition or subtraction. They are asked to convert from the source representation, the vector field plot, to a target representation, which is an electric field lines diagram in Q1.2 and an algebraic expression of the electric field in Q2.2. The students are asked to explain how their target representation is related to the magnitude and direction of the electric field.

The third question (Q1.3 and Q2.3) shows an electric field lines diagram of a non-Coulombic electric field with concentric circular symmetry that decreases with the distance from the center. The diagram does not show any sources of the field, but it explicitly states that the electric field is non-Coulombic. In this question, the task is to convert from the source representation, the electric field lines diagram, to the target representation: an algebraic expression of the electric field in Q1.3 and a vector field plot in Q2.3. Students are asked to explain how their target representation is related to the magnitude and direction of the electric field. This problem may be complicated for students because it shows a non-Coulombic electric field with circular symmetry that decreases with the radius. 
Nonetheless, by the time they participated in the study, both the introductory and the upper-division electricity and magnetism course students had already received explicit instruction on the electric field created by time variations of magnetic fields, mostly on the topic of Faraday's law. Therefore, the presentation of the problem was not new to the students or different from the course contents at both levels.

\section{B. Participants}

We performed a study with 96 engineering physics students from a private Mexican university and 50 students from a Spanish university. We selected the students from Mexico and Spain to observe if there were similar or different tendencies in both groups of participants. We did not aim to compare the performance between groups. From the Mexican participants, we selected introductory and upper-division-level students to observe the similarities and differences between groups of participants who had different levels of expertise. Of the 96 Mexican participants, 64 had finished the introductory electricity and magnetism course (Mex-Intro), and 32 had concluded the upperdivision electromagnetic theory course (Mex-UD). The 50 Spanish students (Spa-UD) took the upper-division electricity and magnetism course in the fall term of 2018 with traditional face-to-face instruction.

The Mex-Intro students took an active learning introductory electricity and magnetism course in the fall semester of 2017, where they used multiple representations. The introductory course covers the main topics of electricity, electric circuits, and magnetism. The course consists of $3 \mathrm{~h}$ of class and $1.5 \mathrm{~h}$ of laboratory sessions per week, with a total of 16 weeks in the semester. It takes place in a SCALE-UP environment. The $3 \mathrm{~h}$ of class are a combination of lectures, peer instruction, problem-solving, and scaffolding activities [49]. The textbook for this course is widely used for teaching introductory physics [48]. In the laboratory sessions, students use Tutorials for Introductory Physics [50].

The Mex-UD students took the same introductory electricity and magnetism course in the fall semester of 2016 and the upper-division electromagnetism course in the fall term of 2017 with traditional instruction. Students enrolled in this course are in the third year of the engineering physics degree. The upper-division course refers to the textbook [51], which is standard for upper-division electromagnetism. The course consists of $3 \mathrm{~h}$ of lecture per week, with a total of 16 weeks in the semester.

The Spa-UD students took the upper-division electricity and magnetism course in the fall term of 2018 with traditional instruction. The course consists of $4 \mathrm{~h}$ of lecture per week, with a total of 15 weeks in the term. The textbook for this course is detailed in Ref. [51]. Students in this course had completed the credits for the first two years and were specifically studying Electromagnetism II in the third year of their degree. Moreover, the third-year physics students had taken an introductory physics course in their first year, which had included the topics described for the introductory electromagnetism syllabus and Electromagnetism I in the second year of their degree $[47,51]$.

\section{Data collection}

We administered the two parts of the test randomly to all students, in such a way that each student answered only one part of the test. The tests were administered in Spanish. We explicitly told students that their performance on the test would not impact their grades. Nevertheless, we encouraged them to answer the test as best as possible and to explain their reasoning. To ensure the students' anonymity, we explicitly asked them not to include personal information, such as their name and last name. The total time to answer the test was $25 \mathrm{~min}$.

\section{Data analysis}

We analyzed the data using a qualitative method to categorize the students' approach to each question and considered the sources of difficulty in Duval's theory (i.e., recognition and conversion). The classification of students into categories was validated by four researchers and Cohen's kappa $(\kappa=0.90)$ as an interrater reliability indicator $[2,5]$. The categories are presented in Table I in a hierarchical order. Category A means that both the recognition and conversion were successful. In categories B and $B^{\prime}$, only the recognition or only the conversion was successful, respectively. In category $\mathrm{C}$, students made an unsuccessful attempt to recognize and convert. In category $\mathrm{D}$, the students did not attempt to recognize and convert, or their answers were incoherent.

Even though we analyzed all questions with the same categories, the meanings for each category were different for each question due to the required type of conversion. To identify what each category conveys for each question, we considered all drawings, equations, and explanations that the students answered. We describe and illustrate the explanations that the students used to support their answers and the most common difficulties that they experienced in recognition and conversion. In this analysis, we did not

TABLE I. Categories based on the primary sources of difficulty in conversion tasks between semiotic representations. Categories follow a hierarchical order in which we identified the students' success of recognition or conversion.

\begin{tabular}{lcc}
\hline \hline Category & Recognition & Conversion \\
\hline A & Successful & Successful \\
B & Successful & Unsuccessful \\
$B^{\prime}$ & Unsuccessful & Successful \\
C & Unsuccessful & Unsuccessful \\
D & Incoherent & Incoherent \\
\hline \hline
\end{tabular}


1. Se tiene una placa no conductora muy grande con carga volumétrica uniforme $\rho$ y altura $d$. En la figura se muestra un corte de la placa.

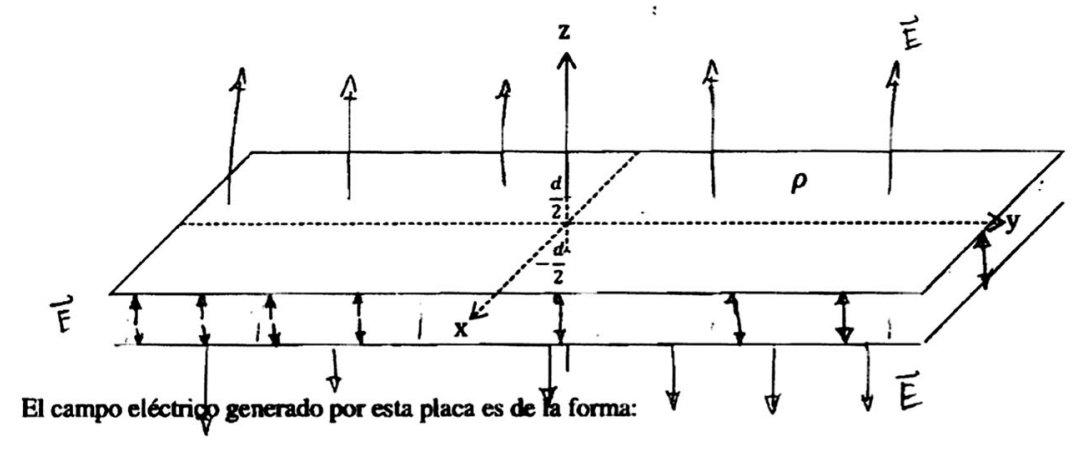

FIG. 3. The text shown in the figure translates to "There is a very large, nonconducting plate with uniform volume charge density $\rho$ and height $d$. The figure shows a section of the plate. The electric field generated by this plate is given by." The full question is found in the Appendix. This example illustrates the answer given by Student Mex-UD 1. This student made proper recognition of the algebraic notation, but a poor conversion to the vector field plot, which classifies the answer into category B for question Q1.1.

focus on category D because it shows a set of incoherent ideas that do not adhere to any model in particular.

\section{The algebraic notation as the source representation: $Q 1.1$ and $Q 2.1$}

In question Q1.1, students were asked to convert from the algebraic notation to the vector field plot. In this question, a successful recognition in categories A and B means that the student interprets the mathematical equation correctly, identifying that the electric field is 0 where $z=0$, its magnitude increases along the $z$ axis inside the plate, and that it is uniform outside the plate. The student also recognizes that the electric field points in the positive direction of $z$ (upward) when $z>0$ and downward when $z<0$. In category $\mathrm{A}$, after recognizing correctly, the students drew vectors that show the relative magnitude and direction of the field in all the different regions. Whereas in category B, they failed to draw vectors correctly, confining the length inside the plate, or not showing that the electric field is uniform outside the plate.

For example, a student from the Mexican university in the Upper-division course (Mex-UD 1), stated that "the field inside the plate changes when passing through 0 , and its magnitude grows as it moves away from the origin. The electric field only has a component in $z$, and outside the plate, it has a constant magnitude at any point, and with opposite directions above and below." We can see from the explanation and the drawing in Fig. 3 that the student made a proper and complete recognition of the characteristics of the electric field in the algebraic notation, but failed to convert to the vector field plot effectively. The vectors inside the plate are confined; it seems like they should not cross the limit of the plate. Neither the vectors inside nor outside are a good representation of the magnitude of the field; inside, they do not represent the variable electric field, while outside, they fail to show that the electric field is uniform.
Mistakes of this sort were grouped under category B. Category $B^{\prime}$ did not emerge in this conversion task. Category $\mathrm{C}$ focuses on unsuccessful recognition and conversion attempts. In this category, we classified those students who did not interpret the mathematical equation correctly. Explicitly, in the region inside the plate, some students stated that the electric field is stronger inside the plate.

In question Q2.1, the physical situation is the same, but students must convert from the algebraic notation to the electric field lines diagram. A successful recognition means that students identify the behavior of the electric field correctly in their explanations, as in Q1.1. A successful conversion in category A means that students draw electric field lines that are equally spaced outside the plate and a variable, increasingly dense set of lines inside the plate. An unsuccessful conversion in category B means that students drew equally spaced field lines outside and inside the plate, as shown in Fig. 4, or they drew a vector field plot instead of field lines. On the other hand, unsuccessful recognition in category $\mathrm{C}$ means that the student explicitly stated that the electric field inside the plate is stronger than outside the plate, as in Q1.1, and drew a vector field plot instead of field lines. Category $B^{\prime}$ did not emerge in these results.

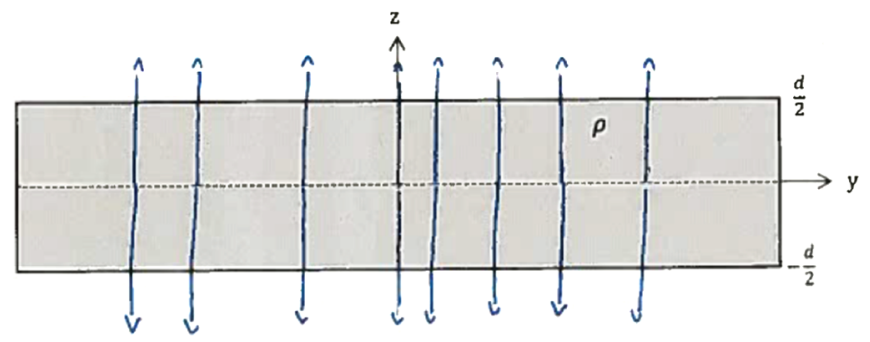

FIG. 4. Student Spa-UD 3 made an adequate recognition of the algebraic notation, but their conversion to electric field lines fails to depict the magnitude of the field correctly. This was therefore classified in category B for question Q2.1. 
The example in Fig. 4 shows an electric field lines diagram that correctly depicts the direction of the field in all regions, but fails to represent the magnitude of the field. The student who drew this diagram made a correct interpretation of the equation: "The orientation of the electric field lines is $k$. In the points where $z$ is null, the electric field is null, and as $z$ grows, the module of $E$ grows until $z=d / 2$. When $z$ is bigger than $d / 2$ or smaller than $-d / 2$, the electric field is constant. For $z>0$, the sense is $k$, and for $z<0$, the sense is $-k$." However, the student's drawing does not show equally spaced lines outside the plate, nor variable density inside. Note that in some countries, one can describe the direction of a vector in terms of orientation and sense, as noted by Nguyen and Meltzer [18]. Answers similar to this were classified as category B because it is clear that students successfully recognized the characteristics of the electric field in the algebraic notation, but did not convert to the electric field lines efficiently.

\section{The vector field plot as the source representation: $Q 1.2$ and $Q 2.2$}

In question Q1.2 the students converted from the vector field plot to the electric field lines representation. Successful recognition in categories A and B means that students correctly identify that the electric field is uniform because all the vectors point in the same direction and have the same length. Regarding the conversion, in category A, students drew electric field lines in the same direction of the vectors, equally spaced, regardless of the vectors that the field lines touched. On the other hand, in category B, we grouped those students that had difficulties with the electric field lines diagram. For example, some students joined the vectors together in continuous lines. In some cases, this is not necessarily incorrect. However, it can be an indicator that students do not draw the electric field lines carefully, considering both the curvature to which the vectors are tangent and the density of the field lines.

An unsuccessful recognition in category $\mathrm{C}$ was that students confused the density of vectors with the magnitude of the field. For example, student Mex-UD 15 stated, "The field lines are in the same direction as the electric field vectors, and their magnitude is bigger where there are more field lines," and drew field lines between the vectors, as shown in Figure 5. Other students who seemed to confuse the density of field lines with the density of vectors would try to "fill the gaps" in the vector plot with more vectors. This category is noteworthy because it gives an insight into the deficiencies in understanding the representations of the electric field, rather than the concept itself.

In question Q2.2, students were asked to create an algebraic expression of the same vector field plot as in Q1.2. In category $A$, the students made a successful recognition, and they expressed the field as a sum of constants in Cartesian unit vectors, relating their answers to

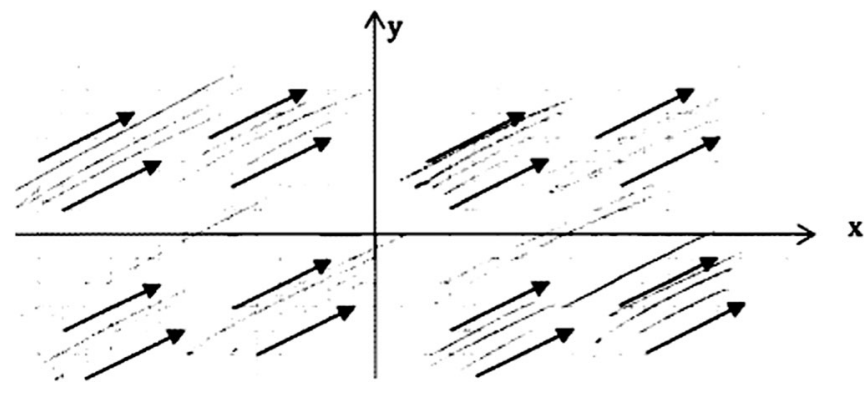

FIG. 5. Student Mex-UD 12 showed confusion in understanding the difference between electric field lines density and vector density. They explained that the electric field is stronger near the vectors, and there should be more electric field lines between them.

the characteristics of the electric field. In category $\mathrm{B}$, the interpretation is also correct, but the student used a different representation system, for example, with a matrix. An unsuccessful recognition and conversion in this question, considered in category $\mathrm{C}$, means that students would express the electric field as a function of variables, either Cartesian or polar when the electric field is shown to be uniform and does not depend on variables.

In this question, category $B^{\prime}$ emerged because some students were able to make a conversion without giving evidence of successful recognition of the electric field in the source representation. It was common for students to express the field as a sum of constants in Cartesian unit vectors without relating their answers to the characteristics of the electric field. For example, student Mex-Intro 11 gave the expression $\vec{E}=4 \hat{i}+2 \hat{j}$, followed by the explanation, "For the direction, I used " $\hat{i}$ " and " $\hat{j}$ ". You can see in the arrows that they have a component in $x$ of 4 units and 2 units in $y$, both positive. All the arrows have these components. Regarding the magnitude, it would be only to calculate the magnitude of the vector."

Mathematically speaking, this is a good explanation of the vector field plot, and the mathematical expression proposed by the student suffices. However, from a physics point of view, this student does not necessarily recognize that this vector field plot is an electric field plot. The student does not explicitly acknowledge that the electric field shown is uniform, nor does he or she make any mention of the electric field in the explanation. This is interesting because it means that students are so familiar with the vector field plot and the algebraic notation that they can move from one to the other without really accessing the physical object, which is the electric field. Of course, we are aware that this emerging category has the limitation that we cannot identify whether or not the student thought of the electric field and only wrote mathematically. Nevertheless, it is useful for this analysis to identify those students who are more comfortable speaking only in mathematical terms and leaving the physical meaning aside. 


\section{The electric field lines diagram as the source representation: $Q 1.3$ and $Q 2.3$}

In question Q1.3, students were asked to convert from an electric field lines diagram to the algebraic notation of the field. In category A, students interpreted the electric field lines accurately and created an algebraic expression that includes proportionality constant, indicates an inverse dependence on the radius, and a counterclockwise direction. In category B, the equation did not indicate proportionality or a counterclockwise direction.

The difficulties in recognition found in this question emerged when students tried to associate the electric field lines diagram with a source. In category $B^{\prime}$, the students would try to associate the field with a magnetic source, writing an expression that used $I$ and/or $\mu_{0}$ as proportionality constants. This would be a correct conversion, but the recognition is unsuccessful because, instead of recognizing the characteristics of the field in the given representation, students recurred to other memoristic resources like the magnetic field produced by a linear electric current.

In category $\mathrm{C}$, students would try to associate with an electric or electromagnetic source. Their conversions included $q$ and/or $\varepsilon_{0}$ as proportionality constants and indicated an inverse dependence on $r^{2}$. Some students did not make a conversion, explaining that the electric field shown could not exist, even though they had already seen non-Coulombian electric fields in the course.

In question Q2.3, students were asked to convert from the electric field lines diagram to the vector field plot. Category A includes those students who interpreted that the electric field decreases radially and that the direction is counterclockwise and drew enough vectors to represent the changes in magnitude and direction. We did not find any students in categories B or $B^{\prime}$. In category $\mathrm{C}$, students made an unsuccessful recognition because they would interpret that the electric field increased radially and draw vectors accordingly. For example, student Mex-UD 5 drew the vectors shown in Fig. 6, correctly interpreting that the

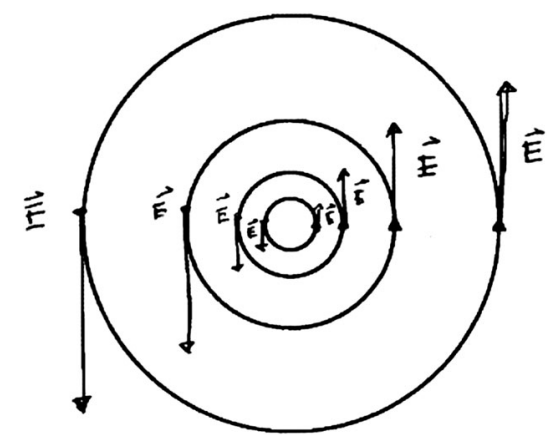

FIG. 6. Student Mex-UD 5 interpreted the direction of the field correctly but the magnitude of the field incorrectly. In this electric field lines diagram, the field decreases with the radius, which is shown through the density of field lines. However, the student represented it as a radially increasing field. direction is tangent to the field lines, but incorrectly interpreting that the magnitude of the field increases radially. In the explanation, the student stated "variable magnitude and variable direction."

\section{RESULTS}

According to the research questions, we present the results in two sections. The first section focuses on the difficulties of recognition and the second section on conversion. In the section of recognition, we present the analyses for two cases where the difficulties of recognition were more evident and relevant to this study, namely, when the electric field lines diagram is the source representation (see Fig. 2, Q1.3 and Q2.3) and the algebraic notation is the target representation (see Fig. 2, Q1.3 and Q2.2). The section of conversion presents a comparison between conversions in opposite directions (see Fig. 2, Q2.2 VS Q1.1, Q1.3 VS Q2.1, and Q1.2 VS Q2.3).

\section{A. Difficulties recognizing the electric field in representations}

\section{Difficulties of recognition: electric field lines as the source representation}

We present the results for the two questions that have the electric field lines diagram as a source representation in Table II. Our results suggest that students who successfully recognize the characteristics of the electric field in the field lines diagram can convert efficiently to the vector field plot and with few difficulties to the algebraic notation. However, the majority of students have problems recognizing the characteristics of the field in the electric field lines diagram, those considered in categories $B^{\prime}, \mathrm{C}$, and D (from Table II, about $70 \%$ for Q1.3 and 73\% for Q2.3 in average). We found that to convert from the electric field lines representation to the algebraic notation (Q1.3), students tried to associate the field lines diagram with a source, either electric or magnetic, instead of interpreting the characteristics of the field from the information given by the representation. This solving strategy is similar to the behavior observed by Campos and Zavala [52] when electricity and magnetism professors interpret an electric field lines diagram. In question Q2.3, the difficulties of recognition are related to the density of field lines since students drew vectors that increase in size from the center. This is further analyzed in Sec. V. B. 2.

\section{Difficulties of recognition: Algebraic notation as the target representation}

In Table III, we present the results for those questions where the algebraic notation is the target representation. It is interesting to analyze these results because category $B^{\prime}$ appeared only when converting from a graphical 
TABLE II. Comparison of results between the questions where the electric field lines diagram is the source representation. The results are shown for introductory and upper-division students in both questions. Mex-Intro and Mex-UD represent the introductory and upperdivision students from the Mexican university, respectively. Spa-UD represents the upper-division students from the Spanish university. We identify the number of students in each category and the corresponding percentage in parentheses. Note that we included an additional row where we add the categories $B^{\prime}, \mathrm{C}$, and $\mathrm{D}$, to facilitate the lecture of the text.

\begin{tabular}{|c|c|c|c|c|c|c|}
\hline \multirow[b]{3}{*}{ Category } & \multicolumn{3}{|c|}{ Q1.3 } & \multicolumn{3}{|c|}{$\mathrm{Q} 2.3$} \\
\hline & Mex-Intro & Mex-UD & Spa-UD & Mex-Intro & Mex-UD & Spa-UD \\
\hline & $n(\%)$ & $n(\%)$ & $n(\%)$ & $n(\%)$ & $n(\%)$ & $n(\%)$ \\
\hline A & 5 (15.6) & $5(31.3)$ & $8(32.0)$ & $9(28.1)$ & $4(25.0)$ & $4(16.0)$ \\
\hline B & $2(6.3)$ & $1(6.3)$ & $0(0.0)$ & $0(0.0)$ & $0(0.0)$ & $3(12.0)$ \\
\hline$B^{\prime}$ & $1(3.1)$ & $2(12.5)$ & $0(0.0)$ & $0(0.0)$ & $0(0.0)$ & $0(0.0)$ \\
\hline $\mathrm{C}$ & $20(62.5)$ & $7(43.7)$ & $10(40.0)$ & $21(65.6)$ & 11 (68.7) & $13(52.0)$ \\
\hline D & $4(12.5)$ & $1(6.3)$ & 7 (28.0) & $2(6.3)$ & $1(6.3)$ & $5(20.0)$ \\
\hline Total & $32(100.0)$ & $16(100.0)$ & $25(100.0)$ & $32(100.0)$ & $16(100.0)$ & $25(100.0)$ \\
\hline$B^{\prime}+C+D$ & $25(78.1)$ & $10(62.5)$ & $17(68.0)$ & $23(71.8)$ & $12(75.0)$ & $18(72.0)$ \\
\hline
\end{tabular}

TABLE III. Comparison of results between questions where the algebraic notation is the target representation. We present the results for both introductory and upper-division students for questions Q1.3 and Q2.2. We identify the number of students in each category and their respective percentages in parentheses.

\begin{tabular}{|c|c|c|c|c|c|c|}
\hline \multirow[b]{4}{*}{ Category } & \multicolumn{6}{|c|}{ Algebraic notation as target representation } \\
\hline & \multicolumn{3}{|c|}{ Q1.3 } & \multicolumn{3}{|c|}{$\mathrm{Q} 2.2$} \\
\hline & Mex-Intro & Mex-UD & Spa-UD & Mex-Intro & Mex-UD & Spa-UD \\
\hline & $n(\%)$ & $n(\%)$ & $n(\%)$ & $n(\%)$ & $n(\%)$ & $n(\%)$ \\
\hline A & $5(15.6)$ & $5(31.3)$ & $8(32.0)$ & $14(43.8)$ & $11(68.8)$ & $15(60.0)$ \\
\hline $\mathrm{B}$ & $2(6.3)$ & $1(6.3)$ & $0(0.0)$ & $1(3.1)$ & $1(6.3)$ & $1(4.0)$ \\
\hline$B^{\prime}$ & $1(3.1)$ & $2(12.5)$ & $0(0.0)$ & 7 (21.9) & $1(6.3)$ & $5(20.0)$ \\
\hline $\mathrm{C}$ & $20(62.5)$ & 7 (43.7) & $10(40.0)$ & $6(18.8)$ & $2(12.4)$ & $3(12.0)$ \\
\hline D & $4(12.5)$ & $1(6.3)$ & $7(28.0)$ & $4(12.5)$ & $1(6.3)$ & $1(4.0)$ \\
\hline Total & $32(100.0)$ & $16(100.0)$ & $25(100.0)$ & $32(100.0)$ & $16(100.0)$ & $25(100.0)$ \\
\hline
\end{tabular}

representation (i.e., field lines or vector plots) to the algebraic notation. This category suggests that students do not need to make a correct or complete recognition of the characteristics of the electric field in the source representation to convert successfully. This suggests that students memorize situations and apply a chug and plug approach, which has been observed in different studies and contexts regarding the algebraic notation [26].

In question Q1.3, students convert from electric field lines to the algebraic notation of the field. The problem of recognition that emerges in category $B^{\prime}$ is an association of the field lines in the diagram with a magnetic source. In this case, since the electric field is non-Coulombic, the mathematical expression that students constructed in category $B^{\prime}$ is adequate because it correctly identifies a proportionality constant (e.g., $\mu_{0}$ ) and an inverse dependence on distance $\left(1 / r^{n}\right)$. However, these students also included in their equations an electric current (usually $I$ o $I_{0}$ ), which is not presented in the problem. This implies that students associated the electric field lines with a circular symmetry to the textbook problem of an outward line of current. This is an incorrect association because students would most probably think about a magnetic field instead of an electric field.

In question Q2.2, students convert from a vector field plot to the algebraic notation. The problem of recognition that emerged in category $B^{\prime}$ is that students correctly indicate a sum of constants in Cartesian coordinates with appropriate unit vectors. However, they did not relate their diagram to the characteristics of the electric field. The students classified under this category might have interpreted the vector plot as an electric field, but since they were not explicit in their answers, it is impossible to know if they were able to identify the characteristics of the field in the representation. The fact that this category emerged implies that students are so familiar with the conversion between these two representations in other contexts that they can convert between them without accessing the object, which implies a memoristic approach in the conversion process. 


\section{B. Difficulties related to the direction of conversion}

Here we present an analysis of the effect that changing the direction of conversion represents for students' performance and their comprehension of the electric field concept. It should be noted that the different directions of conversion are in separate tests, so none of the students performed the conversion in both directions. However, since we administered the tests randomly and because the categories have the same structure, it is possible to compare the results when switching the direction of conversion. The results shown are, therefore, for the groups in general and not for individual students.

\section{Conversions between the algebraic notation and the vector field plot}

Question Q1.1 has the objective to convert from the algebraic notation to the vector field plot, while Q2.2 has the opposite direction of conversion. As shown in Table IV, upper-division students have the same success rate for recognition (categories A and $\mathrm{B}$ ) in both directions (about $75 \%$ ), but the success in conversion (categories $A$ and $B^{\prime}$ ) is higher when converting from vector field plot to algebraic notation (about 75\%) than vice versa (about 25\%). The success rate for recognition is slightly lower for introductory students, but the overall behavior persists (Vector plot to algebraic, $46.8 \%$ vs Algebraic to vector plot, $37.5 \%$ ).

There is a higher tendency to recognize and convert in question Q2.2 than in Q1.1 successfully. Conversely, in Q1.1, students could recognize, but several difficulties arose in their conversions, which indicates that the students had difficulties drawing vector field plots that consider all the characteristics of the field. The most common difficulties observed in this question (Q1.1) were related to representing the magnitude of the field adequately with the length of the arrows. Some students restrained the length of the vectors inside the plate so that they would not cross to the outside region. This is not necessarily incorrect, but the vectors outside the plate need to have similar lengths. We grouped under category B only those students whose outside vectors were considerably larger than the vectors inside the plate. Other students drew vectors outside the plate only at the surfaces, which is not enough to represent that the electric field outside the plate is uniform. Some students combined both difficulties.

\section{Conversion between the vector field plot and electric field lines}

In question Q1.2, students were asked to convert from the vector field plot to electric field lines and, in Q2.3, in the opposite direction. Table V compares the students' performance in this change of direction of conversion. We observe that in both directions students had problems with recognition. However, it is clear from the results that the students' success in converting from electric field lines to the vector field plot relies heavily on successful recognition. Unfortunately, less than $30 \%$ of students recognized all the features of the electric field successfully, which is quite low.

The most common difficulty in Q2.3 was drawing small vectors near the center of the concentric circles and longer vectors as the radius increases (as shown in Fig. 6). This difficulty could mean that the students associate the size of the circles with the intensity of the field, instead of recurring to the density of field lines. An ever-increasing electric field would mean that its intensity tends to infinity with the radius, which is an impossible physical situation. Student SPA-UD 21 seemed to struggle to identify the variation of magnitude in the electric field lines representation and was able to overcome this obstacle by making the physical meaning of the situation. This example is illustrated in Fig. 7. Note that the question does not require the use of algebraic notation, but the student found

TABLE IV. Comparison of results between the algebraic notation and the vector field plot representations. We present the results for both introductory and upper-division students for questions Q1.1 and Q2.2. We identify the number of students in each category and their respective percentages in parentheses. Note that we included two additional rows where we add the categories A with B and $B^{\prime}$, respectively, to facilitate the lecture of the text.

\begin{tabular}{|c|c|c|c|c|c|c|}
\hline \multirow[b]{3}{*}{ Category } & \multicolumn{3}{|c|}{ Q1.1: Algebraic to vector field plot } & \multicolumn{3}{|c|}{ Q2.2 Vector field plot to algebraic } \\
\hline & Mex-Intro & Mex-UD & Spa-UD & Mex-Intro & Mex-UD & Spa-UD \\
\hline & $n(\%)$ & $n(\%)$ & $n(\%)$ & $n(\%)$ & $n(\%)$ & $n(\%)$ \\
\hline A & $7(21.9)$ & $4(25.0)$ & $5(20.0)$ & $14(43.8)$ & $11(68.8)$ & $15(60.0)$ \\
\hline $\mathrm{B}$ & $5(15.6)$ & $8(50.0)$ & $13(52.0)$ & $1(3.1)$ & $1(6.3)$ & $1(4.0)$ \\
\hline$B^{\prime}$ & $0(0.0)$ & $0(0.0)$ & $0(0.0)$ & $7(21.9)$ & $1(6.3)$ & $5(20.0)$ \\
\hline $\mathrm{C}$ & $13(40.6)$ & $3(18.7)$ & $7(28.0)$ & $6(18.8)$ & $2(12.4)$ & $3(12.0)$ \\
\hline $\mathrm{D}$ & $7(21.9)$ & $1(6.3)$ & $0(0.0)$ & $4(12.5)$ & $1(6.3)$ & $1(4.0)$ \\
\hline Total & $32(100.0)$ & $16(100.0)$ & $25(100.0)$ & $32(100.0)$ & $16(100.0)$ & $25(100.0)$ \\
\hline$A+B$ & $12(37.5)$ & $12(75.0)$ & $18(72.0)$ & $15(46.8)$ & $12(75.0)$ & $16(64.0)$ \\
\hline$A+B^{\prime}$ & 7 (21.9) & $4(25.0)$ & $5(20.0)$ & $21(65.5)$ & $12(75.0)$ & $20(80.0)$ \\
\hline
\end{tabular}


TABLE V. Comparison of results between the vector field plot and the electric field lines representations. We present the results for both introductory and upper-division students for questions Q1.2 and Q2.3. We identify the number of students in each category and their respective percentages in parentheses.

\begin{tabular}{|c|c|c|c|c|c|c|}
\hline \multirow[b]{3}{*}{ Category } & \multicolumn{3}{|c|}{ Q1.2: Vector field plot to electric field lines } & \multicolumn{3}{|c|}{ Q2.3: Electric field lines to vector field plot } \\
\hline & Mex-Intro & Mex-UD & Spa-UD & Mex-Intro & Mex-UD & Spa-UD \\
\hline & $n(\%)$ & $n(\%)$ & $n(\%)$ & $n(\%)$ & $n(\%)$ & $n(\%)$ \\
\hline A & $6(18.7)$ & $5(31.2)$ & $7(28.0)$ & $9(28.1)$ & $4(25.0)$ & $4(16.0)$ \\
\hline $\mathrm{B}$ & $11(34.4)$ & $1(6.3)$ & $7(28.0)$ & $0(0.0)$ & $0(0.0)$ & $3(12.0)$ \\
\hline$B^{\prime}$ & $0(0.0)$ & $0(0.0)$ & $0(0.0)$ & $0(0.0)$ & $0(0.0)$ & $0(0.0)$ \\
\hline $\mathrm{C}$ & $6(18.8)$ & $6(37.5)$ & $7(28.0)$ & $21(65.6)$ & $11(68.7)$ & $13(52.0)$ \\
\hline $\mathrm{D}$ & $9(28.1)$ & $4(25.0)$ & $4(16.0)$ & $2(6.3)$ & $1(6.3)$ & $5(20.0)$ \\
\hline Total & $32(100.0)$ & $16(100.0)$ & $25(100.0)$ & $32(100.0)$ & $16(100.0)$ & $25(100.0)$ \\
\hline
\end{tabular}

it useful to convert to the algebraic notation as part of their sensemaking.

As shown in Fig. 7, the student Spa-UD 21 did not recur to the density of field lines to identify that the electric field decreases with the radius. The student was able to identify with an algebraic expression that the magnitude varies with the radius and, through applying physical principles and sense making, identified that the magnitude should decrease, drawing vectors that decreased their length. The lengths of the vectors are not very well defined, which is consistent with the students' explanation, "I do not know the way in which the magnitude varies; I assume that it decreases with the distance." Instead of using the visual characteristics of the electric field lines' representation to relate the magnitude of the field to the separation between the field lines, the student recurred to the physical meaning of this particular situation to conclude that the electric field decreases with the radius. This finding is noteworthy. It suggests that misinterpretation of the electric field lines diagram can be overcome by the successful recognition of physical principles and sensemaking.

In the opposite direction, there are fewer difficulties of recognition, but more of conversion, as expected, because drawing electric field line diagrams accurately is a complicated task. The most common difficulty in Q1.2 was joining vectors in continuous lines, which compromises the density of field lines and derives in an incorrect representation of the magnitude of the field. It is compelling that the most common difficulties in both directions of conversion are related directly to dissociation between the density of

3. La figum eepresents las lincas de un campo eléctrico no Coulombiano.

a) Dibuja sobre la figura varios vectores que describan el campo eléctrico en distintas posiciones.
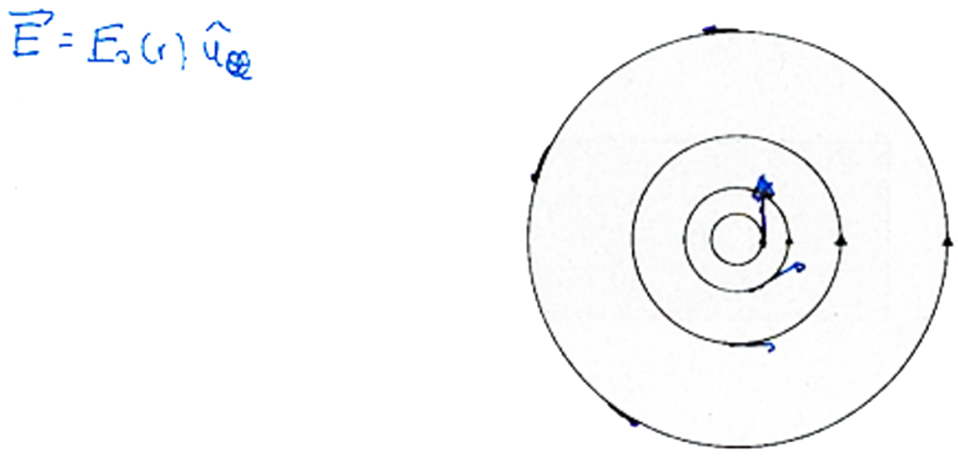

b) Explica cómo se relacion a u dibujo con el campo ciectrico de la figura, poniencio atención en las caracteristicas del campo: midido, dirección y sentido.

FIG. 7. The text of the question in the figure translates to "The figure represents the electric field lines of a non-Coulombic electric field. (a) Draw several vectors that correspond to the electric field in the figure. (b) Explain how your drawing is related to the electric field shown in terms of the features of the electric field, namely, magnitude and direction." The full question is found in the Appendix. This example illustrates the answer given by Student Spa-UD 21. The students' explanation in point (b), translated from Spanish, reads: "In principle, since it [the field] is non-Coulombic, it [the magnitude] does not need to reduce with the squared distance; nonetheless, I believe that it must somehow reduce with the distance from the center, because I do not think it makes physical sense, an electric field that tends to infinity at infinity. So, even though I do not know the way in which the magnitude varies, I assume that it decreases with the distance [from the center]. Regarding the direction, it is tangent to the line at each point with the orientation given by the arrowhead." 
TABLE VI. Comparison of results between the algebraic and the electric field line representations. We present the results for both introductory and upper-division students for questions Q1.3 and Q2.1. We identify the number of students in each category and their respective percentages in parentheses.

\begin{tabular}{|c|c|c|c|c|c|c|}
\hline \multirow[b]{3}{*}{ Category } & \multicolumn{3}{|c|}{ Q1.3: Electric field lines to algebraic } & \multicolumn{3}{|c|}{ Q2.1: Algebraic to electric field lines } \\
\hline & Mex-Intro & Mex-UD & Spa-UD & Mex-Intro & Mex-UD & Spa-UD \\
\hline & $n(\%)$ & $n(\%)$ & $n(\%)$ & $n(\%)$ & $n(\%)$ & $n(\%)$ \\
\hline A & $5(15.6)$ & $5(31.3)$ & $8(32.0)$ & $2(6.3)$ & $0(0.0)$ & $1(4.0)$ \\
\hline B & $2(6.3)$ & $1(6.3)$ & $0(0.0)$ & $11(34.4)$ & $12(75.0)$ & $18(72.0)$ \\
\hline$B^{\prime}$ & $1(3.1)$ & $2(12.5)$ & $0(0.0)$ & $0(0.0)$ & $0(0.0)$ & $0(0.0)$ \\
\hline $\mathrm{C}$ & $20(62.5)$ & $7(43.7)$ & $10(40.0)$ & $15(46.8)$ & $3(18.7)$ & $4(16.0)$ \\
\hline $\mathrm{D}$ & $4(12.5)$ & $1(6.3)$ & $7(28.0)$ & $4(12.5)$ & $1(6.3)$ & $2(8.0)$ \\
\hline Total & $32(100.0)$ & $16(100.0)$ & 25 (100.0) & $32(100.0)$ & $16(100.0)$ & $25(100.0)$ \\
\hline
\end{tabular}

field lines and the magnitude of the electric field. This difficulty has been previously reported in the literature by several studies $[3,20,40]$, which reaffirms the relevance of this finding.

\section{Conversion between electric field lines diagram and algebraic notation}

Table VI shows students' performance when converting between the electric field lines diagram and the algebraic notation. Between these two representations, the direction of conversion has a significant effect on the students' abilities of recognition and conversion. In question Q1.3, the students who successfully recognized the characteristics of the field were able to convert successfully, but the success rates were low (similar to Q2.3, analyzed in the previous section). It should be noted that question Q1.3 presents the two problems of recognition that were discussed before, namely, failure to recognize the density of field lines as the magnitude of the electric field and the emergence of category $B^{\prime}$, where students were able to convert to the algebraic notation without fully interpreting the source representation.

When the direction of conversion changes, the students who successfully recognized the characteristics of the field did not make successful conversions. In question Q2.1, students had many difficulties to convert from the algebraic notation directly to the electric field lines diagram. Only three students out of 73 who answered this question drew electric field lines. The remaining students who did a successful recognition drew a vector field plot and, therefore, became classified in category B. The students' explanations in this category lead to three possible implications: (i) students do not differentiate between electric field lines diagrams and vector field plots efficiently; (ii) both the electric field lines diagram and the vector field plot serve the purpose of giving an overview of the electric field, so for ease of construction, the students chose to draw a vector field plot (we have noted before that drawing electric field lines diagrams is a complicated task), and (iii) students need to first construct a vector field plot in order to draw an electric field lines diagram. However, in this case, they only did the intermediate step of constructing the vector field plot.

\section{DISCUSSION}

The theory of registers of semiotic representation creates a direct link between students' abilities of recognition and conversion in several registers of representation and their conceptual understanding of the abstract concept that is represented. As Duval [33] affirms, to achieve conceptual understanding, it is necessary to have synergy between two or even three different representation systems. We presented our students with several conversion tasks between three different registers of representation of the electric field, namely, the algebraic notation, the vector field plot, and the electric field lines diagram. From our results, we can analyze and compare the synergy that the students have between these three different registers of representation of the electric field. We focus the discussion on this matter because it allows us to associate the students' representational flexibility with their conceptual understanding of the electric field. The tendencies of synergy between representation systems presented in this discussion are similar for all the groups of participants, which implies that they persist through background (Mexico and Spain) and level of expertise (introductory and upper-division).

We found that students have good synergy converting from the algebraic notation to the vector field plot and vice versa. The majority of students can recognize the characteristics of the field in both representations successfully; only a small portion of students had difficulties of recognition, failing to relate the mathematical concept of the vector field to the electric field, while still being able to make a successful conversion. Even though recognition is high, they do have some difficulties drawing the vector field plot (Q1.1), which are similar to those reported by Bollen, Van Kampen, Baily, Kelly, and De Cock [36]. Precisely, the arrow length did not match the magnitude of the field when students tried to restrict vectors inside the plate. Also, they would only draw one set of vectors outside the plate, which 
is not enough to represent that the electric field is uniform in that region. We acknowledge that the classification of students under this category has an intrinsic limitation since we cannot identify if a student has correct reasoning and understanding of the representation, but decides to draw only one set of vectors for simplicity or practicality. Some students might have the necessary recognition and conversion skills to be considered in the A category, but we cannot identify them objectively. When working with these two representations, students should be encouraged to relate the algebraic notation to the characteristics of the electric field and to identify all the key positions that are necessary to represent the electric field in a vector field plot fully.

We found that students do not have good synergy in the conversions that involve the electric field lines diagram. Between the vector field plot and the electric field lines diagram, the results are discouraging because, even though both representations are visual, there seems to be poor synergy between them. This problem is associated with an inability to recognize the density of field lines as the magnitude of the field in the electric field lines representation. This source of difficulty emerged in both directions of conversion. Interpreting the density of field lines as the magnitude of the electric field is a difficulty that has been reported in the literature by several studies $[3,12,20,36,40,53]$. We also found that there is not good synergy between algebraic notation and electric field lines. When doing the conversion from algebraic notation to the electric field lines diagram, students would achieve proper recognition, but most of them drew a vector field plot instead. In the opposite direction, students had difficulties with recognition because they tried to identify a source of the field, as some instructors do when interpreting electric field lines [52]. Interestingly, when converting from electric field lines to the algebraic notation, students were able to identify an inverse dependency on the radius, but when they converted from field lines to the vector field plot, they identified a direct dependency on the radius [54]. This behavior is different from the behavior reported by Ref. [36] because, in their study, students answered first the conversion to a vector field plot, and then they converted to the algebraic notation; this might have led students to drag the same reasoning into the algebraic expression.

It is important to note that students are experiencing a learning process in which they aim to develop from novices to experts [29]. Within this process of conceptual understanding, we see that some students have difficulties that agree with those reported in the literature $[3,12,20,36,40,53]$. These difficulties are often associated with the recognition of the characteristics of the electric field within the semiotic representations. Furthermore, we observed some difficulties of conversion, which are mostly driven by the direction of the conversion between registers of representation, in agreement with Duval's theory. For students to achieve the expected conceptual understanding, they would need to be able to convert in both directions efficiently, such as experts do. This behavior is not observed in the conversions that involve electric field lines, either as the source or the target representation.

\section{CONCLUSION}

Our objective in this study was to learn how students understand the concept of the electric field through the use of multiple representations of this physical quantity. To achieve this objective, we focused on the students' abilities to recognize the main characteristics of the electric field (namely, magnitude and direction) in three representations: the algebraic notation, the vector field plot, and the electric field lines diagram, and how they convert between them. We analyzed data within the framework of Duval's theory of semiotic representations. Physics instructors should consider that good synergy between two or three representations is necessary for student conceptual understanding of abstract properties, as proposed by Duval [33]. It is, therefore, of utmost importance to dedicate time and practice to use several representations for the concept of an electric field to promote a conceptual understanding of this physical quantity.

We approached the question of how students recognize the characteristics of the electric field in different semiotic representations. We found that, in general, students have more difficulties recognizing the electric field lines representation than the vector field plot and the algebraic notation of the field. For the electric field lines representation, the difficulties of recognition are that the students try to associate the diagram with a source of an electric field, instead of interpreting the features of the representation. Other difficulties are related to not recognizing the density of field lines as the magnitude of the electric field. For the vector field plot, a few students had difficulties recognizing that the electric field is uniform in the problem that they analyzed. Other students would relate the density of vectors in the field plot to the intensity of the electric field. For the algebraic notation, some students had difficulties interpreting the electric field inside the plate. Overall, the difficulties of recognition that we found are related to interpreting the magnitude of the electric field, rather than the direction.

We also approached the question of how students convert between the three mentioned semiotic representations of the electric field. We found that electric field lines were quite challenging for students to make conversions, both as a source and as a target representation. As a source representation, many students were not able to make a conversion because the difficulties in recognition were their first obstacle. As a target representation, many students avoided making electric field lines diagrams and chose to draw vector field plots instead. For the vector field plot representation, some students confined the vectors to the 
borders of the object (in this case, a plate), and other students did not consider enough points to represent a uniform electric field. For the algebraic notation of the field, the difficulties of conversion were related to using other representations in the symbolic or arithmetic family, such as matrices and coordinate axis, instead of the algebraic vector notation.

Our results are limited by the characteristics of each of our two different cohorts of students, Mexican and Spanish. Even though we share the same language, cultural and educational contexts are different. We have seen that, regardless of our differences, our students share some of the difficulties. Our contribution is focused on the results that are shared, regardless of the background. The characteristics of the groups of students and the difficulties that we have seen are similar to those of other cohorts of students, particularly American and European. The textbooks that we use are comparable to those used in American and European universities, both in content and level. The educational culture is also similar; we may have some teachers who still use traditional instruction, while other teachers include active learning. Some of our results are comparable with results done in different parts of the world with different groups of students see, for example, Refs. $[2,3,12,20,36,40,53]$. Therefore, we may assume that the results could be similar in other American and European contexts. We think that this may be of interest to researchers in the area of electricity and magnetism, specifically on the learning of the concept of an electric field, which sets the basis for Maxwell's relations. From our findings, teachers and researchers in the area might be more aware of the difficulties that some conversion tasks may represent for students, which adds to the discussion of how the concept of an electric field is taught and learned at the university level.

Based on Duval's theory of semiotic representations, our study explores the connection between understanding the three widely used representations and the understanding of the electric field concept. Our contribution is novel because it analyzes that a conceptual understanding must be related to the conversion capacity of the three representations. This relationship is essential because traditional teaching bases its teaching strategy on field representations as a way of "facilitating" student understanding. Several studies have pointed out that the electric field lines representation is difficult for students, which matches with our results. Even though this representation is useful for experts to have an overall visualization of the electric field, we question if the way they are traditionally taught in the introductory courses is helpful for science and engineering students, or if it hinders their understanding of the concept of an electric field. To answer this concern, we would need to conduct several studies where electric field lines are approached actively, explicitly, consistently, and with proper scaffolding during the course. In this way, we could identify if the students' difficulties with this representation are due to the properties of the representation itself or if they can be solved through active and explicit instruction. We recommend that instructors pay special attention to how they approach this representation in class and be very explicit about how the density of field lines represents the magnitude of the field.

\section{ACKNOWLEDGMENTS}

We thank Dr. J. I. Barragues for his contributions to our discussions about Duval's theory of semiotic representations. We also acknowledge the partial financial support of the School of Engineering and Sciences of Tecnologico de Monterrey, the Basque Government Project No. PIBA IT1349-19, of the Spanish Government MINECO EDU2015-65359-P. We acknowledge the financial and technical support of Writing Lab, TecLabs, Tecnologico de Monterrey, Mexico, in the production of this work.

\section{APPENDIX: QUESTIONNAIRES ADMINISTERED TO STUDENTS}

The following are the two questionnaires that were administered to students. Each student presented only one of the questionnaires.

Questionnaire 1

1. There is a very large, nonconducting plate with uniform volume charge density $\rho$ and height $d$. Figure 8 shows a section of the plate.

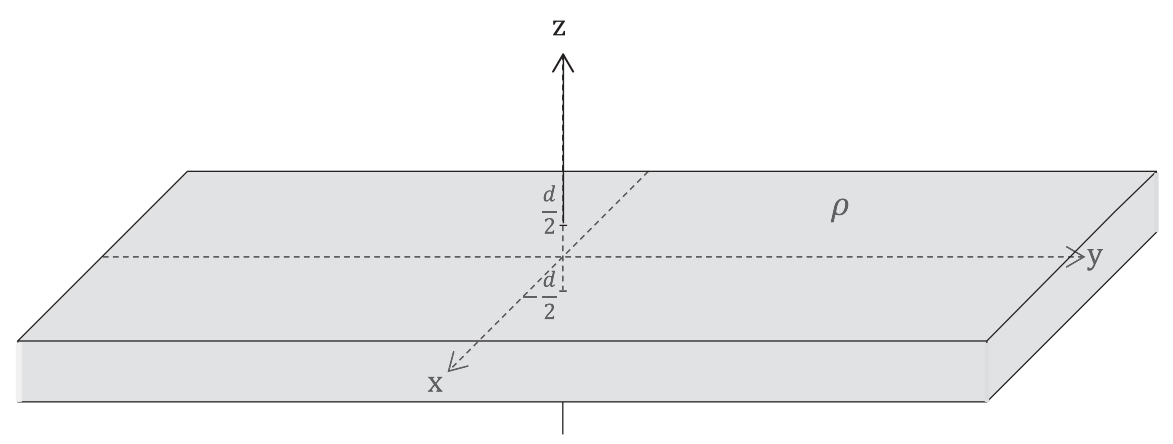

FIG. 8. Schematic of a very large, nonconducting plate used in question Q1.1. 


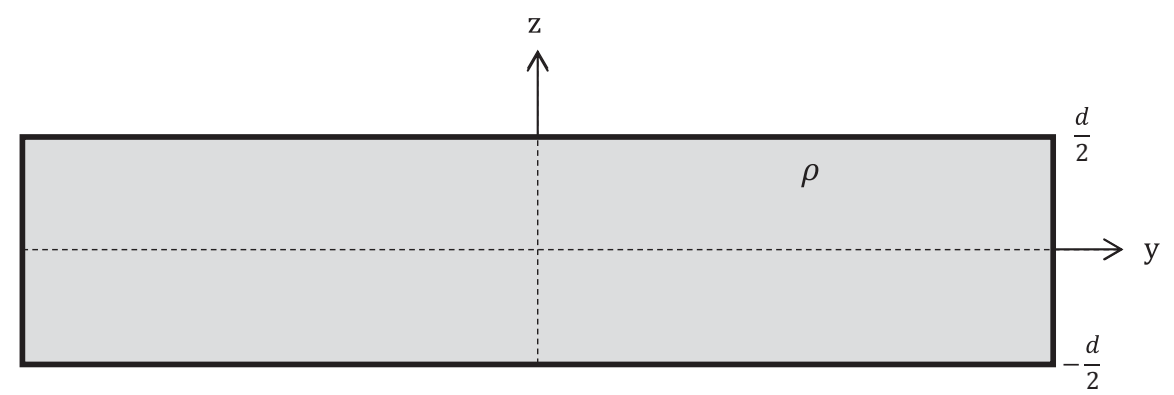

FIG. 9. Schematic of a frontal cut of a very large, nonconducting plate used in question Q1.1.

The electric field generated by this plate is given by

$$
\vec{E}=\left\{\begin{array}{cc}
\frac{\rho}{\varepsilon_{0}} z \hat{k} ; & -\frac{d}{2}<z<\frac{d}{2} \\
-\frac{\rho}{\varepsilon_{0}} \frac{d}{2} \hat{k} ; & z<-\frac{d}{2} \\
\frac{\rho}{\varepsilon_{0}} \frac{d}{2} \hat{k} ; & z>\frac{d}{2}
\end{array}\right.
$$

a. Figure 9 shows a frontal cut of the plate. Draw several vectors that correspond to the electric field inside and outside the plate.

b. Explain how your drawing is related to the function of the electric field in terms of the features of the electric field: magnitude and direction.

2. There is an electric field such as the one shown in Fig. 10 at plane $x-y$

a. Draw on the figure the electric field lines for the electric field shown.

b. Explain how your drawing is related to the electric field shown in terms of the features of the electric field: magnitude and direction.

3. Figure 11 represents the electric field lines of a nonCoulombic electric field.

(a) Write a mathematical expression to describe the electric field in the figure.

(b) Explain how your expression is related to the electric field shown in terms of the features of the electric field: magnitude and direction.

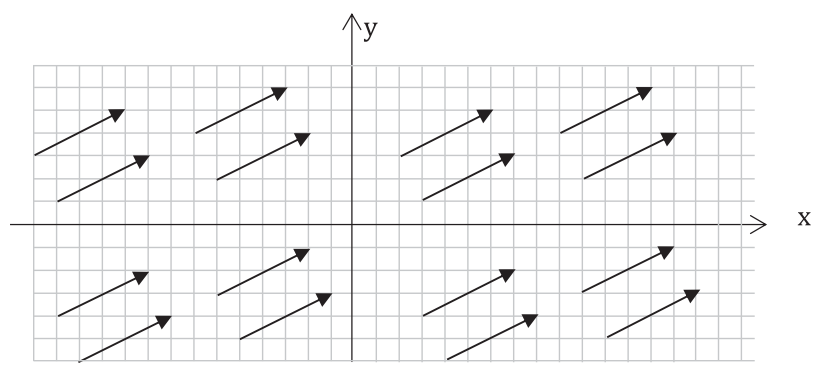

FIG. 10. Vector field plot of a uniform electric field used in question Q1.2.

\section{Questionnaire 2}

1. There is a very large, nonconducting plate with uniform volume charge density $\rho$ and height $d$. Figure 12 shows a section of the plate.

The electric field generated by this plate is given by

$$
\vec{E}=\left\{\begin{array}{cc}
\frac{\rho}{\varepsilon_{0}} z \hat{k} ; & -\frac{d}{2}<z<\frac{d}{2} \\
-\frac{\rho}{\varepsilon_{0}} \frac{d}{2} \hat{k} ; & z<-\frac{d}{2} \\
\frac{\rho}{\varepsilon_{0}} \frac{d}{2} \hat{k} ; & z>\frac{d}{2}
\end{array}\right.
$$

a. Figure 13 shows a frontal cut of the plate. Draw electric field lines that correspond to the electric field inside and outside the plate.

b. Explain how your drawing is related to the function of the electric field in terms of the features of the electric field: magnitude and direction.

2. There is an electric field such as the one shown in Fig. 14 at plane $x-y$

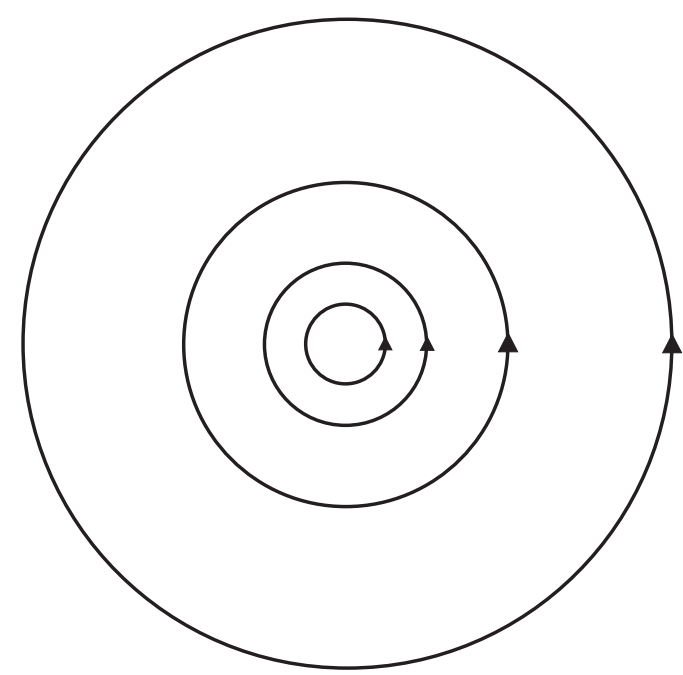

FIG. 11. Vector field plot of a uniform electric field used in question Q1.3. 


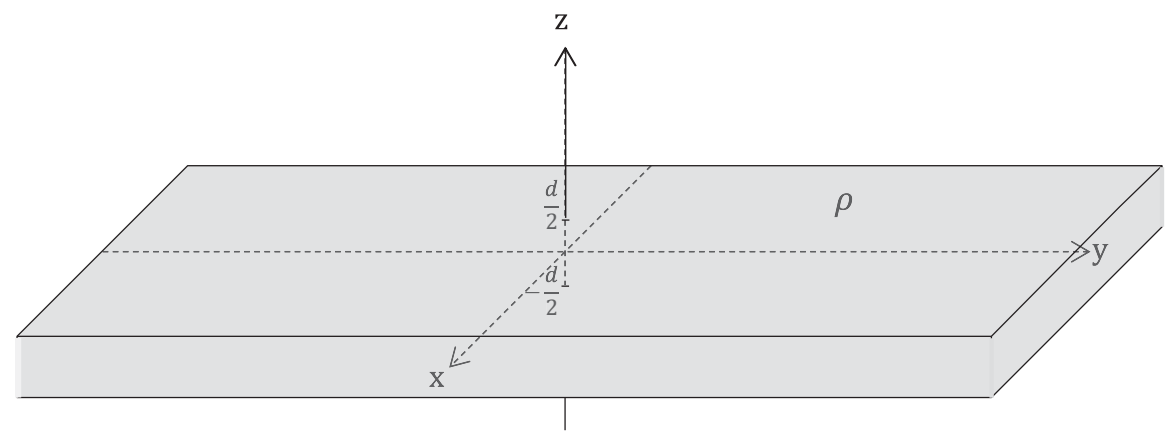

FIG. 12. Schematic of a very large, nonconducting plate used in question Q2.1.

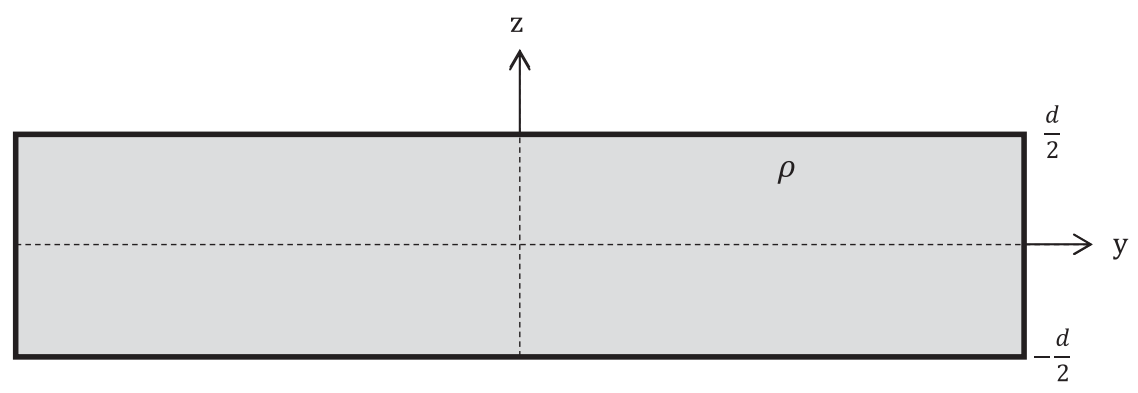

FIG. 13. Schematic of a frontal cut of a large, nonconducting plate used in question Q2.1.

a. Write a mathematical expression for the electric field shown.

b. Explain how your expression is related to the electric field shown in terms of the features of the electric field: magnitude and direction.

3. Figure 15 represents the electric field lines of a nonCoulombic electric field.

(a) Draw several vectors that correspond to the electric field in the figure.

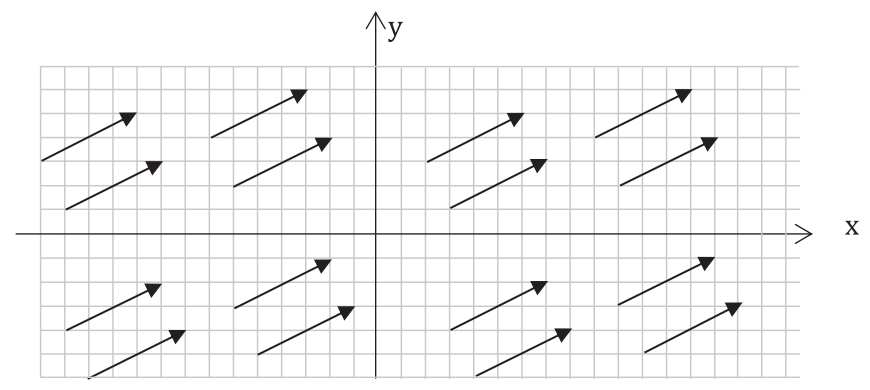

FIG. 14. Vector field plot of a uniform electric field used in question Q2.2. (b) Explain how your drawing is related to the electric field shown in terms of the features of the electric field: magnitude and direction.

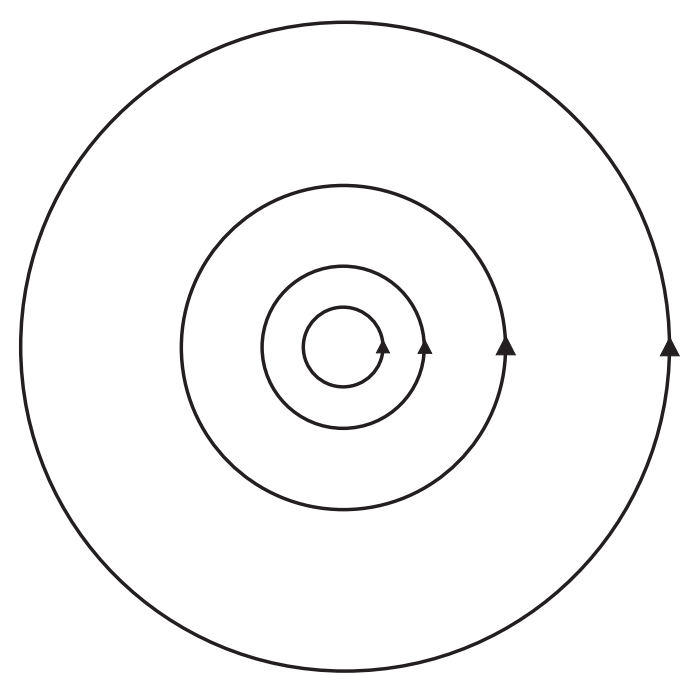

FIG. 15. Vector field plot of a uniform electric field used in question Q3.3. 
[1] C. Furió and J. Guisasola, Difficulties in learning the concept of electric field, Sci. Educ. 82, 511 (1998).

[2] K. Zuza, P. van Kampen, M. De Cock, T. Kelly, and J. Guisasola, Introductory university physics students' understanding of some key characteristics of classical theory of the electromagnetic field, Phys. Rev. Phys. Educ. Res. 14, 020117 (2018).

[3] M. Saarelainen, A. Laaksonen, and P. E. Hirvonen, Students' initial knowledge of electric and magnetic fields-more profound explanations and reasoning models for undesired conceptions, Eur. J. Phys. 28, 51 (2007).

[4] C. Furió, J. Guisasola, J. M. Almudí, and M. Ceberio, Learning the electric field concept as oriented research activity, Sci. Educ. 87, 640 (2003).

[5] A. Leniz, K. Zuza, and J. Guisasola, Students' reasoning when tackling electric field and potential in explanation of dc resistive circuits, Phys. Rev. Phys. Educ. Res. 13, 010128 (2017).

[6] P. Barniol, E. Campos, and G. Zavala, La prueba conceptual de electricidad y magnetismo: análisis de confiabilidad y estudio de las dificultades más frecuentes, Enseñanza de las ciencias 36, 167 (2018).

[7] X. Bohigas and C. Periago, Modelos mentales alternativos de los alumnos de segundo curso de ingeniería sobre la Ley de Coulomb y el Campo Eléctrico, Revista Electrónica de Investigación Educativa 12, 1 (2010).

[8] L. Viennot and S. Rainson, Students' reasoning about the superposition of electric fields, Int. J. Sci. Educ. 14, 475 (1992).

[9] S. Rainson, G. Tranströmer, and L. Viennot, Students' understanding of superposition of electric fields, Am. J. Phys. 62, 1026 (1994).

[10] C. Singh, Student understanding of symmetry and Gauss's law of electricity, Am. J. Phys. 74, 923 (2006).

[11] A. Garza and G. Zavala, Contrasting students' understanding of electric field and electric force, AIP Conf. Proc. 1513, 142 (2013).

[12] J. Li and C. Singh, Investigating and improving introductory physics students' understanding of the electric field and superposition principle, Eur. J. Phys. 38, 055702 (2017).

[13] V. Albe, P. Venturini, and J. Lascours, Electromagnetic concepts in mathematical representation of physics, J. Sci. Educ. Technol. 10, 197 (2001).

[14] R. E. Pepper, S. V. Chasteen, S. J. Pollock, and K. K. Perkins, Observations on student difficulties with mathematics in upper-division electricity and magnetism, Phys. Rev. ST Phys. Educ. Res. 8, 010111 (2012).

[15] B. R. Wilcox, M. D. Caballero, R. E. Pepper, and S. J. Pollock, Upper-division student understanding of Coulomb's law: Difficulties with continuous charge distributions, AIP Conf. Proc. 1513, 418 (2013).

[16] L. Bollen, P. van Kampen, and M. De Cock, Students' difficulties with vector calculus in electrodynamics, Phys. Rev. ST Phys. Educ. Res. 11, 020129 (2015).

[17] L. Bollen, P. van Kampen, C. Baily, and M. De Cock, Qualitative investigation into students' use of divergence and curl in electromagnetism, Phys. Rev. Phys. Educ. Res. 12, 020134 (2016).
[18] N. L. Nguyen and D. E. Meltzer, Initial understanding of vector concepts among students in introductory physics courses, Am. J. Phys. 71, 630 (2003).

[19] E. Gire and E. Price, Arrows as anchors: An analysis of the material features of electric field vector arrows, Phys. Rev. ST Phys. Educ. Res. 10, 020112 (2014).

[20] S. Törnkvist, K. A. Pettersson, and G. Tranströmer, Confusion by representation: On student's comprehension of the electric field concept, Am. J. Phys. 61, 335 (1993).

[21] Y. Cao and B. M. Brizuela, High school students' representations and understandings of electric fields, Phys. Rev. Phys. Educ. Res. 12, 020102 (2016).

[22] M. C. Pocoví, The effects of a history-based instructional material on the students' understanding of field lines, J. Res. Sci. Teach. 44, 107 (2007).

[23] M. C. Pocovi and F. N. Finley, Historical evolution of the field view and textbook accounts, Sci. Educ. 12, 387 (2003).

[24] P. B. Kohl and N. D. Finkelstein, Representational format, student choice, and problem solving in physics, AIP Conf. Proc. 790, 121 (2005).

[25] P. B. Kohl and N. D. Finkelstein, Student representational competence and self-assessment when solving physics problems, Phys. Rev. ST Phys. Educ. Res. 1, 010104 (2005).

[26] P. B. Kohl and N. D. Finkelstein, Effects of representation on students solving physics problems: A fine-grained characterization, Phys. Rev. ST Phys. Educ. Res. 2, 010106 (2006).

[27] M. De Cock, Representation use, and strategy choice in physics problem solving, Phys. Rev. ST Phys. Educ. Res. 8, 020117 (2012).

[28] T. Fredlund, J. Airey, and C. Linder, Exploring the role of physics representations: an illustrative example from students sharing knowledge about refraction, Eur. J. Phys. 33, 657 (2012).

[29] M. T. Chi, R. Glaser, and E. Rees, Expertise in problem solving (No. TR-5). (Pittsburgh Univ Pa Learning Research And Development Center, Pittsburgh, PA, 1981).

[30] P. B. Kohl and N. D. Finkelstein, Patterns of multiple representation use by experts and novices during physics problem solving, Phys. Rev. ST Phys. Educ. Res. 4, 010111 (2008).

[31] C. Singh, When physical intuition fails, Am. J. Phys. 70, 1103 (2002).

[32] R. J. Dufresne, W. J. Gerace, P. T. Hardiman, and J. P. Mestre, Constraining novices to perform expertlike problem analyses: Effects on schema acquisition, J. Learn. Sci. 2, 307 (1992).

[33] R. Duval, A cognitive analysis of problems of comprehension in a learning of mathematics, Educ. Stud. Math. 61, 103 (2006).

[34] J. Guisasola, Teaching and learning electricity: the relations between macroscopic level observations and microscopic level theories, in International Handbook of Research in History, Philosophy and Science Teaching, edited by M. R. Matthews (Springer Netherlands, Dordrecht, 2014).

[35] P. Barniol and G. Zavala, Test of understanding of vectors: A reliable multiple-choice vector concept test, Phys. Rev. ST Phys. Educ. Res. 10, 010121 (2014). 
[36] L. Bollen, P. van Kampen, C. Baily, M. Kelly, and M. De Cock, Student difficulties regarding symbolic and graphical representations of vector fields, Phys. Rev. Phys. Educ. Res. 13, 020109 (2017).

[37] R. D. Knight, The Vector Knowledge of Beginning Physics Students, Phys. Teach. 33, 74 (1995).

[38] S. Flores, S. E. Kanim, and C. H. Kautz, Student use of vectors in introductory mechanics, Am. J. Phys. 72, 460 (2004).

[39] J. M. Hawkins, J. R. Thompson, M. C. Wittmann, E. C. Sayre, and B. W. Frank, Students' responses to different representations of a vector addition question, AIP Conf. Proc. 1289, 165 (2010).

[40] E. Campos and G. Zavala, A look into students' interpretation of electric field lines, in Handbook of Research on Driving STEM Learning with Educational Technologies, edited by M.S. Ramírez-Montoya (IGI Global, Hershey, PA, 2017).

[41] O. R. Battaglia, C. Fazio, and R. M. Sperandeo-Mineo, An inquiry-based approach to Maxwell distribution: a case study with engineering students, Eur. J. Phys. 34, 975 (2013).

[42] C. Fazio, O. R. Battaglia, and B. Di Paola, Investigating the quality of mental models deployed by undergraduate engineering students in creating explanations: The case of thermally activated phenomena, Phys. Rev. ST Phys. Educ. Res. 9, 020101 (2013).

[43] T. Fredlund, C. Linder, J. Airey, and A. Linder, Unpacking physics representations: Towards an appreciation of disciplinary affordance, Phys. Rev. ST Phys. Educ. Res. 10, 020129 (2014).

[44] S. Ceuppens, J. Deprez, W. Dehaene, and M. De Cock, Design and validation of a test for representational fluency of 9th grade students in physics and mathematics: The case of linear functions, Phys. Rev. Phys. Educ. Res. 14, 020105 (2018).
[45] D. Halliday, R. Resnick, and K. S. Krane, Physics (Wiley, New York, 1992), Vol. 2.

[46] R. A. Serway and J. W. Jewett, Physics for Scientists and Engineers with Modern Physics (Cengage Learning EMEA, Boston, MA, 2008).

[47] P. A. Tipler and G. Mosca, Physics for Scientists and Engineers (W. H. Freeman and Company, New York, 2008).

[48] H. D. Young and R. A. Freedman, University Physics with Modern Physics, Technology update, 13th ed. (AddisonWesley, Reading, MA, 2012).

[49] G. Zavala, The design of activities based on cognitive scaffolding to teach physics, in Upgrading Physics Education to Meet the Needs of Society, edited by M. Pietrocola (Springer International Publishing, Switzerland, 2019), pp. 169-179.

[50] L. C. McDermott and P. S. Shaffer, Tutoriales Para Física Introductoria (Pearson Education, Buenos Aires, 2001).

[51] D. J. Griffiths, Introduction to Electrodynamics (Cambridge University Press, Cambridge, England, 2017).

[52] E. Campos and G. Zavala, Exploring instructors' interpretation of electric field lines, in Proceedings of the 2017 Physics Education Research Conference, Cincinnati, OH, edited by L. Ding, A. Traxler, and Y. Cao (AIP, New York, 2017), p. 76.

[53] E. Campos, G. Zavala, K. Zuza, and J. Guisasola, Electric field lines: The implications of students' interpretation on their understanding of the concept of electric field and the superposition principle, Am. J. Phys. 87, 660 (2019).

[54] E. Campos, G. Zavala, K. Zuza, and J. Guisasola, Students' conversion from electric field line diagrams to other representations, in Proceedings of the 2019 Physics Education Research Conference, Provo, UT, edited by Y. Cao, S. Wolf, and M. B. Bennett (AIP, New York, 2019). 